\title{
Multiferroic bismuth ferrite: Perturbed angular correlation studies on its ferroic $\alpha-\beta$ phase transition
}

\author{
G. Marschick $\odot,{ }^{1,2}$ J. Schell $\odot,{ }^{2,3}$ B. Stöger $\odot,{ }^{1}$ J. N. Gonçalves $\odot,{ }^{4}$ M. O. Karabasov, ${ }^{3}$ D. Zyabkin $\odot,{ }^{5}$ A. Welker $\odot,{ }^{2}$ \\ M. Escobar C., ${ }^{3}$ D. Gärtner, ${ }^{6}$ I. Efe ${ }^{7}{ }^{7}$ R. A. Santos ${ }^{8},{ }^{8}$ J. E. M. Laulainen $\odot,{ }^{9}$ and D. C. Lupascu $\circledast^{3}$ \\ ${ }^{1} X$-Ray Center, Vienna University of Technology, 1040 Vienna, Austria \\ ${ }^{2}$ European Organization for Nuclear Research CERN, CH-1211 Geneva, Switzerland \\ ${ }^{3}$ Institute for Materials Science and Center for Nanointegration Duisburg-Essen (CENIDE), \\ University of Duisburg-Essen, 45141 Essen, Germany \\ ${ }^{4}$ Departmento de Física and CICECO, Universidade de Aveiro, 3810-193 Aveiro, Portugal \\ ${ }^{5}$ Chair Materials for Electronics, Institute of Materials Science and Engineering, \\ and Institute of Micro and Nanotechnologies MacroNano ${ }^{\circledR}$, TU Ilmenau, 98693 Ilmenau, Germany \\ ${ }^{6}$ Institut für Materialphysik, AG Wilde, Universität Münster, 48149 Münster, Germany \\ ${ }^{7}$ Department of Materials, ETH Zürich, 8092 Zürich, Switzerland \\ ${ }^{8}$ Hyperfine Interactions Group - IPEN University of São Paulo, São Paulo, São Paulo, Brazil \\ ${ }^{9}$ Department of Materials Science and Metallurgy, University of Cambridge, CP3 OFS Cambridge, United Kingdom
}

(Received 12 May 2020; revised 13 October 2020; accepted 14 December 2020; published 29 December 2020)

\begin{abstract}
Work of numerous research groups has shown different outcomes of studies of the transition from the ferroelectric $\alpha$-phase to the high temperature $\beta$-phase of the multiferroic, magnetoelectric perovskite Bismuth Ferrite $\left(\mathrm{BiFeO}_{3}\right.$ or $\left.\mathrm{BFO}\right)$. Using the perturbed angular correlation (PAC) method with ${ }^{111 m} \mathrm{Cd}$ as the probe nucleus, the $\alpha$ to $\beta$ phase transition was characterized. The phase transition temperature, the change of the crystal structure, and its parameters were supervised with measurements at different temperatures using a six detector PAC setup to observe the $\gamma-\gamma$ decay of the ${ }^{111 m} \mathrm{Cd}$ probe nucleus. The temperature dependence of the hyperfine parameters shows a change in coordination of the probe ion, which substitutes for the bismuth site, forecasting the phase transition to $\beta$-BFO by either increasing disorder or formation of a polytype transition structure. A visible drop of the quadrupole frequency $\omega_{0}$ at a temperature of about $T_{c} \approx 820^{\circ} \mathrm{C}$ indicates the $\alpha-\beta$ phase transition. For a given crystal symmetry, the DFT-calculations yield a specific local symmetry and electric field gradient value of the probe ion. The Pbnm ( $\beta$-BFO) crystal symmetry yields calculated local electric field gradients, which very well match our experimental results. The assumption of other crystal symmetries results in significantly different computed local environments not corresponding to the experiment.
\end{abstract}

DOI: 10.1103/PhysRevB.102.224110

\section{INTRODUCTION}

Significant work on multiferroic materials started to take place through Soviet research in the late 1950s. Generally, a multiferroic can be described as a material that exhibits a combination of at least two of the following ferroic forms of ordering, namely, ferromagnetism (which here includes every kind of magnetic appearance with a macroscopic magnetic moment $M$ plus antiferromagnetism), ferroelectricity, and ferroelasticity, in the same phase [1]. Great focus lies on materials which show a combination of ferromagnetic and ferroelectric properties, as the simultaneous appearance of these forms opens new ways of exploiting material properties like electrically controlled magnetization and vice versa [2].

Published by the American Physical Society under the terms of the Creative Commons Attribution 4.0 International license. Further distribution of this work must maintain attribution to the author(s) and the published article's title, journal citation, and DOI.
Wide fields of application are promised in spintronic and data storage devices as well as in sensoric and actuatoric systems [3]. In this aspect, among all discovered multiferroics, Bismuth Ferrite (also referred to as BFO), owing to its high antiferromagnetic Néel temperature of $T_{N}=370^{\circ} \mathrm{C}$ and high ferroelectric Curie temperature of $T_{c} \approx 825^{\circ} \mathrm{C}$ [4], turned out to be a very promising candidate because it could be trained to exhibit a magnetoelectric effect even at room temperature. The $\alpha$-phase of BFO appears in a rhombohedral structure with the cell parameters $a=5.58 \AA$ and $c=13.87 \AA$ in the hexagonal settings at room temperature. It is a quasicubic canted perovskite structure of the $\mathrm{LiNbO}_{3}$ type [4]. The $\alpha$-phase exists up to $820^{\circ} \mathrm{C}$ where $\mathrm{BFO}$ shows a phase transition to the $\beta$-phase, which is accompanied by a sudden contraction of the unit cell volume. Its character still is the subject of numerous discussions and is mentioned to appear in cubic, trigonal, and even tetragonal structures, as can be seen in Fig. 1 [5-11]. Eventually, Arnold et al. [9] suggested the $\beta$-phase to appear in the orthorhombic Pbnm space group with the cell parameters $a=5.61 \AA, b=5.64 \AA$, and $c=7.97 \AA$. To characterize the $\alpha-\beta$ phase transition in BFO, we applied 


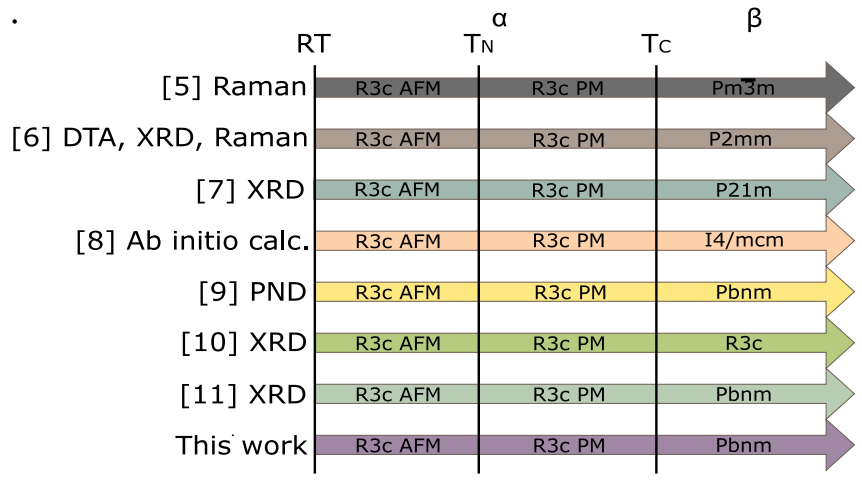

FIG. 1. Summary of selected works addressing the crystallographic settings of the $\beta$-phase of BFO.

a combination of two fundamentally different experimental methods. Whereas perturbed angular correlation (PAC) is a local method, providing information on the nuclear environments, i.e., of one or two unit cells distance to the probe ion, $\mathrm{x}$-ray diffraction (XRD) is a macroscopic method, unveiling details about the crystal structure over extended crystal regions. All PAC measurements were conducted at the isotope mass separator on-line (ISOLDE) facilities [12,13] at CERN in Geneva, Switzerland. XRD measurements were done at the X-Ray Center at the Vienna University of Technology in Vienna, Austria. Using a probe ion in an ionic crystal lattice allows to investigate the very local probe environment. In the isochemical case, e.g., using Mössbauer spectroscopy on ${ }^{57} \mathrm{Fe}$ in an iron containing a compound like $\mathrm{BiFeO}_{3}$, reflects the status of the crystal itself [14]. Using an isovalent but chemically different ion will induce mechanical distortions of the probe, which typically is of different ionic size, but no electrical effect is expected. Depending on the kind of electronic shell, also an isovalent ion may interfere with bonding, e.g., if in the lattice magnetically coupled $d$-orbitals (or $f$-orbitals) partake in bonding and a probe is introduced which does not provide the same $d$-orbitals. Then additional distortions arise due to the unmatched partially filled $d$-orbitals. For a heterovalent probe ion additional distortions may arise due the electrostatic interaction. For solid solutions this induces local electric potentials and may attract other defects like oxygen vacancies. For large concentrations beyond the solid solution range, crystal phase transitions may be induced [15]. In ferroelectrics such probe defects are associated with yet another neighboring defect, typically an oxygen vacancy [16]. It is thus of fundamental interest to understand the role of such defects also from a local perspective. In a first-order phase transition a thermal hysteresis arises at this very phase transition [17]. Bismuth ferrite is a material with high ferroelectric Curie temperature and a strongly first-order phase transition into its paraelectric high temperature phase ( $\beta$-phase). It is thus a prime candidate to investigate the local probe environments at phase transitions. Due to the high temperature of the phase transition, it is also close to the temperature where $\mathrm{BiFeO}_{3}$ decomposes into $\mathrm{Bi}_{2} \mathrm{Fe}_{4} \mathrm{O}_{9}$ under liberation of the volatile $\mathrm{Bi}_{2} \mathrm{O}_{3}$ [18]. For a significant local probe study, a short-lived isotope is advantageous at high radioactive decay rate to keep measurement times as short as possible. As we showed in another work of Schell et al., long-lived PAC probes like ${ }^{181} \mathrm{Hf}$ will suffer from significant changes of the sample during measurement at high temperature and are thus not suitable for this temperature range [19]. As the structural polar ordering of $\mathrm{BiFeO}_{3}$ is constituted by the lone pair $6 s$-electrons of $\mathrm{Bi}$, the discussion on its contribution to crystal structure transformation has been ongoing [20]. Using a local probe, such instabilities can be monitored locally. Additional density functional theory (DFT) calculations can give hints on the structural ordering from a local perspective. Experimentally establishing a relation of such local order to the macroscopic crystal phase structure is intended in this work. ${ }^{111 m} \mathrm{Cd}$ is a short-lived and excellent PAC isotope. As will be discussed later, it occupies the Bi-site and thus represents a heterovalent probe ion in the crystal sublattice, which generates the ferroelectric ordering. It is thus directly exposed to the changes of lone pair orientation in the unit cell, which yields a change in symmetry of the site in case of nonalignment at instability points.

\section{EXPERIMENTAL DETAILS}

The polycrystalline $\mathrm{BiFeO}_{3}$ samples were synthesized at the University of Duisburg-Essen from a stoichiometric mixture of finely powdered bismuth oxide $\left(\mathrm{Bi}_{2} \mathrm{O}_{3}, 99.9 \%\right.$ Acros Organics) and iron oxide $\left(\mathrm{Fe}_{2} \mathrm{O}_{3}, 99.99 \%\right.$ Alfa Aesar). Following a calcination process for 3 hours at $820^{\circ} \mathrm{C}$, the powder was pressed to pellets and sintered for six hours under air atmosphere again at $820^{\circ} \mathrm{C}$. The samples were shipped to the ISOLDE/CERN facility, where, to perform the PAC measurements, the Cadmium isotope ${ }^{111 m} \mathrm{Cd}$ was implanted at the ISOLDE GLM beamline with an accelerator voltage of $30 \mathrm{keV}$. According to simulations with the SRIM software package [21], the implantation depth distribution shows a maximum at around $116 \AA$. The PAC measurements were done immediately after implantation in the facilities of the ISOLDE Solid State Physics Collaboration [13] using a fully digital six detector spectrometer [22]. The furnace with an included sample holder allowed measurements at all desired temperature points. All measurements were performed at temperatures reached from below. No PAC measurement was done on cooling due to the time limits in the experiment. PAC, being a hyperfine interaction technique, allows the investigation of the electromagnetic fields interacting with a nuclear probe located in the material $[23,24]$. The probe nucleus in its excited state decays to its ground state via the sensitive intermediate state through a $\gamma-\gamma$ cascade decay. Through an angular correlation between the first and the second $\gamma$ ray, distinguished through their energies and detection times, hyperfine interactions between the probe nuclei and their surrounding atomic structure can be monitored. A fixed detector, which detects a first quantum $\gamma_{1}$, defines a reference axis, conventionally designated as the $z$-axis. A number of nuclei with their initial spin $I_{i}$ aligned to this direction is selected. This nuclear alignment leads to an anisotropic radiation of $\gamma_{2}$ with regard to $\gamma_{1}$ [25]. If the probe nucleus quadrupole momentum is disturbed through an electric field gradient (EFG) while being in the intermediate state, its spin $I$ will experience a torque which forces a spin-precession around the before-referenced $z$-axis. Caused by this precession, the 
population of the intermediate state sublevels changes with time, which again affects the spacial anisotropy of the $\gamma_{1}-\gamma_{2}$ correlation. An electric field gradient is caused by charge distributions which deviate from an ideal spherically symmetric arrangement around the probe atom. It is defined as the second spacial derivative of the electrostatic potential. Through a diagonalization of the interaction Hamiltonian, rotating the tensor into its principal component coordinate system and the use of the Laplace equation, the EFG can be described by its largest component $V_{z z}$ and the asymmetry parameter

$$
\eta=\frac{V_{x x}-V_{y y}}{V_{z z}}
$$

only. The quadrupole interaction frequency is calculated through

$$
\omega_{q}=\frac{e Q V_{z z}}{4 I(2 I-1) \hbar},
$$

where $e$ is the electron charge, $Q$ the quadrupole moment of the intermediate state of the probe nucleus, $I$ its angular momentum, and $\hbar$ the reduced Planck's constant. For an anisotropy coefficient $\eta$ of zero, the lowest detectable substate-transition frequency is

$$
\omega_{0}=k \omega_{q},
$$

with $k=6$ for half-integer values of spin momentum, like the $I=5 / 2$ level of our probe isotope ${ }^{111 m} \mathrm{Cd}$. The higher-ordered frequencies are calculated through $\omega_{n}=n \omega_{0}$. If $\eta$ is different from zero, the transition frequencies $\omega_{n}$ are functions of $\omega_{q}$ and $\eta$ itself:

$$
\begin{gathered}
\omega_{1}=2 \sqrt{3} \alpha \omega_{q} \sin \left(\frac{1}{3} \arccos (\beta)\right), \\
\omega_{2}=2 \sqrt{3} \alpha \omega_{q} \sin \left(\frac{1}{3}[\pi-\arccos (\beta)]\right), \\
\omega_{3}=2 \sqrt{3} \alpha \omega_{q} \sin \left(\frac{1}{3}[\pi+\arccos (\beta)]\right),
\end{gathered}
$$

with

$$
\alpha=\sqrt{\frac{28\left(3+\eta^{2}\right)}{3}},
$$

and

$$
\beta=\frac{80\left(1-\eta^{2}\right)}{\alpha^{3}} .
$$

These transition frequencies represent the transitions between the substate levels created through the hyperfine splitting of the intermediate state. For a $I=5 / 2$ state and a pure quadrupole moment, the intermediate state is split into three sublevels $E_{Q}(M)$, resulting in three transition frequencies $\omega_{n}$ visible in the perturbation function [Eqs. (4) to (6)]. The normalized values of the sublevel energy states $E_{Q}(M)$ as well as of the transition frequencies $\omega_{n}$ and their change with rising asymmetry parameter $\eta$ are shown in Figs. 2 and 3. Following Gerdau [26], the theoretical perturbation factor for the fit of polycrystalline samples is described by

$$
G_{k k}(\eta, t)=\sum_{n=0}^{3} s_{k n}(\eta) \cos \left[\omega_{n}\left(\eta, V_{z z}\right) t\right] \exp \left(-\delta \omega_{n} t\right),
$$

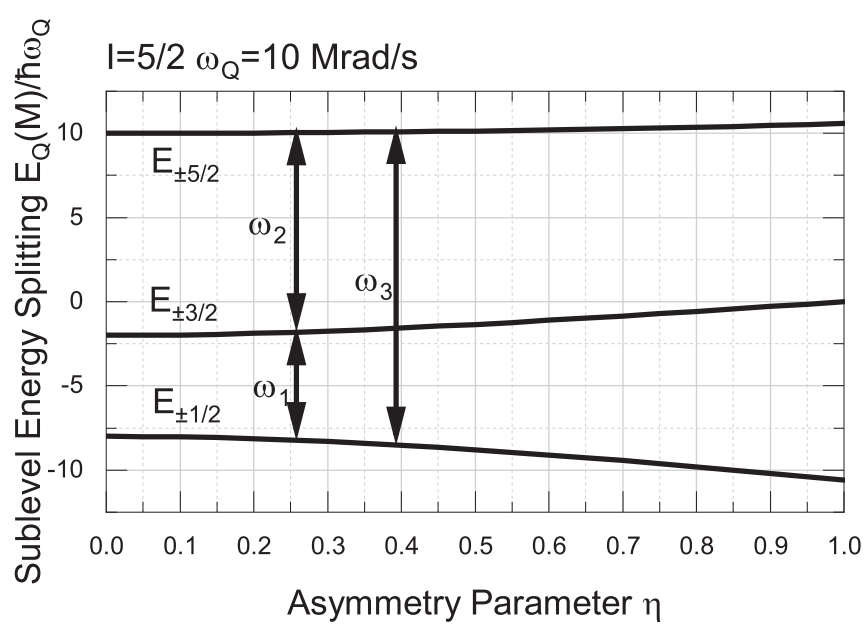

FIG. 2. Change of sublevel energies $E_{Q}(M)$ with increasing asymmetry parameter. Reference values only valid for $I=5 / 2$.

with $\delta$ being the Lorentzian EFG distribution width and $s_{k n}(\eta)$ being weight functions depending on the asymmetry parameter $\eta$. Exact formulas for the weight functions $s_{k n}(\eta)$ for isotopes with an intermediate state of $I=5 / 2$ can also be found in Friedsam [27]. The perturbation function of the $\gamma-\gamma$ angular correlation is finally represented by the anisotropy function

$$
\begin{aligned}
R(t) & =A_{k k} G_{k k}(t) \\
& =A_{k k} \sum_{n=0}^{3} s_{k n}(\eta) \cos \left[\omega_{n}\left(\eta, V_{z z}\right) t\right] \exp \left(-\delta \omega_{n} t\right),
\end{aligned}
$$

with $A_{k k}$ being correlation coefficients depending on the nuclear cascade and $G_{k k}$ containing all information about the EFG [23,27-29].

\section{RESULTS AND DISCUSSION}

\section{A. Simulation results}

As mentioned before, the published data on the $\alpha-\beta$ phase transition temperature and the structure of the $\beta$-phase

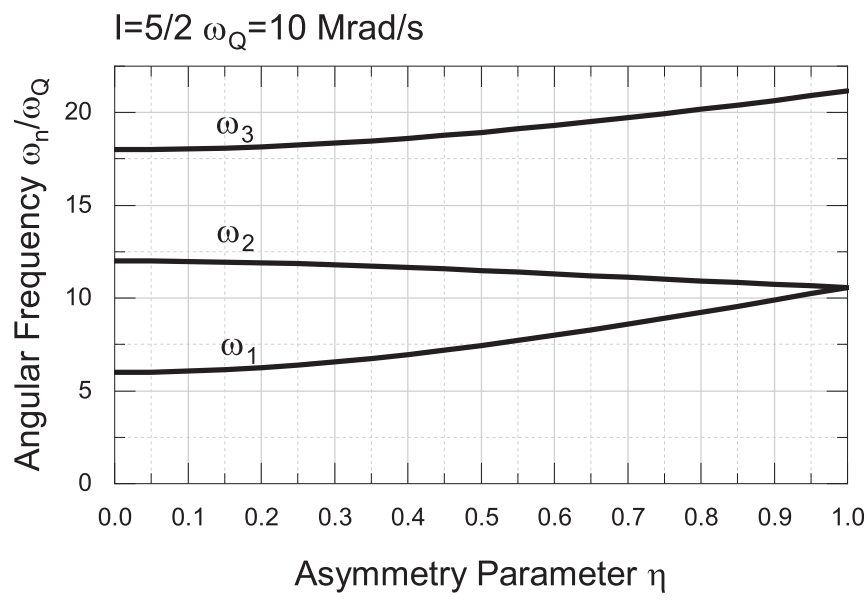

FIG. 3. Resulting change of angular frequencies $\omega_{n}$ calculated with Eqs. (4) to (6). Reference values only valid for $I=5 / 2$. 
TABLE I. Simulated values of $V_{z z}$ and $\Delta H$ as well as the calculated value of $\omega_{0}$. The range of the results of $\omega_{0}$ is caused by the uncertainity of $Q$.

\begin{tabular}{llcccc}
\hline \hline \multicolumn{1}{c}{ System } & & $V_{z z}^{\mathrm{DFT}}\left[\frac{10^{21} V}{m^{2}}\right]$ & $\eta^{\mathrm{DFT}}[1]$ & $\Delta H^{\mathrm{DFT}}[\mathrm{eV}]$ & $\omega_{0}^{\mathrm{DFT}}[\mathrm{Mrad} / \mathrm{s}]$ \\
\hline \multirow{2}{*}{$\alpha$-BFO (FM/AFM) } & $\mathrm{Cd} @ \mathrm{Bi}$ & $5.72 / 5.37$ & $0 / 0$ & $8.4 / 2.6$ & $80.3-86.8 / 75.4-81.5$ \\
& $\mathrm{Cd} @ \mathrm{Fe}$ & $5.46 / 4.07$ & $0 / 0$ & $8.8 / 5.5$ & $76.7-82.9 / 57.1-61.8$ \\
$\beta$-BFO (FM/AFM) & $\mathrm{Cd} \mathrm{@} \mathrm{Bi}$ & $-7.51 /-6.88$ & $0.39 / 0.26$ & $4.2 / 1$ & $105.4-113.97 / 96.57-104.41$ \\
& $\mathrm{Cd} @ \mathrm{Fe}$ & $2.37 / 8.43$ & $0.87 / 0.18$ & $5.4 / 2.2$ & $33.3-35.9 / 118.33-127.93$ \\
$\mathrm{Bi}_{2} \mathrm{Fe}_{4} \mathrm{O}_{9}$ (FM) & $\mathrm{Cd} \mathrm{@} \mathrm{Bi}$ & 9.99 & 0.12 & - & $140.22-151.61$ \\
& $\mathrm{Cd} @ \mathrm{Fe}$ & 7.99 & 0.84 & - & $112.15-121.25$ \\
\hline \hline
\end{tabular}

contradict. The crystal structure of the perovskite BFO offers two different sites for the implanted ${ }^{111 m} \mathrm{Cd}$ probe atom, namely the A-site, substituting the bismuth site and the B-site, substituting iron. To determine the most favorable substitution site as well as to set benchmarks for verification of the PAC results, DFT simulations were done using the Vienna Ab Initio Simulation Package (VASP) program [30]. The employed approach was the projector augmented wave method with a general gradient approximation using the $5 d^{10} 6 s^{2} 6 p^{3}$ valence configuration for $\mathrm{Bi}, 2 s^{2} 2 p^{4}$ configuration for $\mathrm{Fe}, 3 p^{6} 3 d^{6} 4 s^{2}$ configuration for $\mathrm{O}$, and the $4 d^{10} 5 s^{2}$ configuration for $\mathrm{Cd}$. For the $\alpha$-phase $(Z=6)$ calculations, a $2 \times 2 \times 1$ supercell in the hexagonal setting, with chemical formulas $\mathrm{CdBi}_{23} \mathrm{Fe}_{24} \mathrm{O}_{72}$ for $\mathrm{Cd}$ substituting $\mathrm{Bi}$ and $\mathrm{Bi}_{24} \mathrm{CdFe}_{23} \mathrm{O}_{72}$ for $\mathrm{Cd}$ substituting $\mathrm{Fe}$ was used. We used a $k$-point grid of $4 \times 4 \times 3$ for the calculation. The $\beta$-phase $(Z=4)$ calculations were also done within a $2 \times 2 \times 1$ supercell with the formulas $\mathrm{CdBi}_{15} \mathrm{Fe}_{16} \mathrm{O}_{48}$ for $\mathrm{Cd}$ substituting the $\mathrm{Bi}$-site and $\mathrm{Bi}_{16} \mathrm{CdFe}_{15} \mathrm{O}_{48}$ for $\mathrm{Cd}$ substituting the Fe-site with a $k$-point grid of $5 \times 5 \times 4 . \mathrm{Bi}_{2} \mathrm{Fe}_{4} \mathrm{O}_{9}$ $(Z=2)$ is a secondary phase detected in the $\mathrm{x}$-ray diffraction measurements with space group Pbam and lattice parameters $a=7.9826 \AA, b=8.49636 \AA$, and $c=6.04298 \AA$. With this cell the distance between $\mathrm{Cd}$ impurities $(c \approx 6 \AA)$ may be small, so we used a $2 \times 1 \times 2$ supercell where the smallest distance between $\mathrm{Cd}$ impurities increases to the lattice parameter $b \approx 8.5 \AA$ ( $k$-point grid of $3 \times 2 \times 4)$. The $k$-point densities were similar considering the supercell sizes. For all calculations the energy cutoff was $450 \mathrm{eV}$. The self-consistent calculations stopped for energy differences between two steps smaller than $10^{-5} \mathrm{eV}$. Other precision settings were set by the VASP tag PREC $=$ ACCURATE. The atomic positions are optimized to minimize the forces below a threshold of $0.01 \mathrm{eV} / \AA$. The $U$-parameters in use were a $\mathrm{PBE}$ approximation with Coulomb repulsion $U_{\text {eff }}=3 \mathrm{eV}$ applied to $\mathrm{Fe}-d$ electrons [31]. The electric field gradient $V_{z z}$, the anisotropy parameter $\eta$, and the formation energy $\Delta H$ were simulated for $\mathrm{Cd}$ substituting the $\mathrm{Bi}$, as well as the $\mathrm{Fe}$ sites. The quadrupole interaction frequency $\omega_{0}$ was then calculated with the use of formulas 2, 3, and 4 with the simulated values of $V_{z z}, \eta$, and $Q=0.641$ (25) barn [32]. Even though BFO is paramagnetic at our measurement temperatures, the paramagnetic order is very difficult to simulate. Temperature was not taken into account in the simulations. The paraelectric phase was not simulated with molecular dynamics as the computational cost would have been out of proportion considering the outcome. As a workaround, all simulations were done for ferromagnetic (FM) and $G$-type antiferromagnetic (AFM) magnetic spin or- der to determine the EFG dependence on magnetic ordering in general. Simulation results and the calculated values of $\omega_{0}$ of the $\alpha$-phase, the $\beta$-phase and an additional ternary phase, $\mathrm{Bi}_{2} \mathrm{Fe}_{4} \mathrm{O}_{9}$, which will be explained in Sec. III D, can be found in Table I. As the results of the $\alpha$-, and the $\beta$-phases show, the formation energy $\Delta H$ is lower in both the FM and the AFM order in the simulated system of $\mathrm{Cd}$ substituting the $A$-site. Thus for the dynamics explained in this work, a $\mathrm{Cd}$ substitution on the Bi site is energetically more favorable than a substitution on the Fe site, which is backed by the findings of Gebhardt [33]. We also calculated the local effect of two other structures which have been proposed for the $\beta$ phase, considering $\mathrm{Cd}$ substitutional at $\mathrm{Bi}$. For the $R \overline{3} c$ structure, $V_{z z}=$ $18.87 \times 10^{21} \mathrm{~V} / \mathrm{m}^{2}$ and $\eta=0$ were obtained at the Cd site, substitutional at $\mathrm{Bi}$. The corresponding $\omega_{0} \approx 250 \mathrm{Mrad} / \mathrm{s}$ is much higher than experimental values, while $\eta=0$ also does not agree with the high $\eta$ measured in the $\beta$ phase. For the $P 2_{1} / m$ structure, a large supercell calculation $(3 \times 2 \times 3$ of the original $P 2_{1} / m$ ) was used. The obtained EFG at $\mathrm{Cd}$ is $V_{z z}=-24.8 \times 10^{21} \mathrm{~V} / \mathrm{m}^{2}, \eta=0.37$. In this case we used a smaller energy cutoff of $400 \mathrm{eV}$, and the forces are smaller than $4 \mathrm{mRy} /($ bohr radius), a slightly less accurate calculation, due to the large computational time required. Nevertheless, the result should already have qualitatively converged for $V_{z z}$, and the corresponding $\omega_{0} \approx 350 \mathrm{Mrad} / \mathrm{s}$ is very far the from experimental value, allowing us to conclude that this is not the measured configuration.

\section{B. PAC results}

Figure 4 shows the experimental $R(t)$ functions as well as fast fourier transformation (FFT) data with fits at measurement temperatures between $800^{\circ} \mathrm{C}$ and $850^{\circ} \mathrm{C}$. The FFT analysis is helpful to visualize the $R(t)$ functions through the transition frequencies $\omega_{n}$ and their ratio. The connection to the asymmetry parameter $\eta$ (see Fig. 3) of the containing EFGs is directly visible.

As mentioned in Secs. III A and III C, the Cd probe ion substitutes the Bi site within the BFO sample material, leading to sensitivity to changes in its nearest surrounding, which is set up by oxygen atoms forming coordination polyhedra around the $\mathrm{Bi}(\mathrm{Cd})$ atom. The change from a sixfold coordination in the $\alpha$-phase to an eightfold coordination in the $\beta$-phase results from a change of the Bi-site symmetry. An ideal, nondistorted perovskite structure with point group $P m \overline{3} \mathrm{~m}$ shows a 12 -fold coordination on the $A$-site and a 6 -fold coordination on the $B$-site. In the case of BFO though, the perovskite structure is 


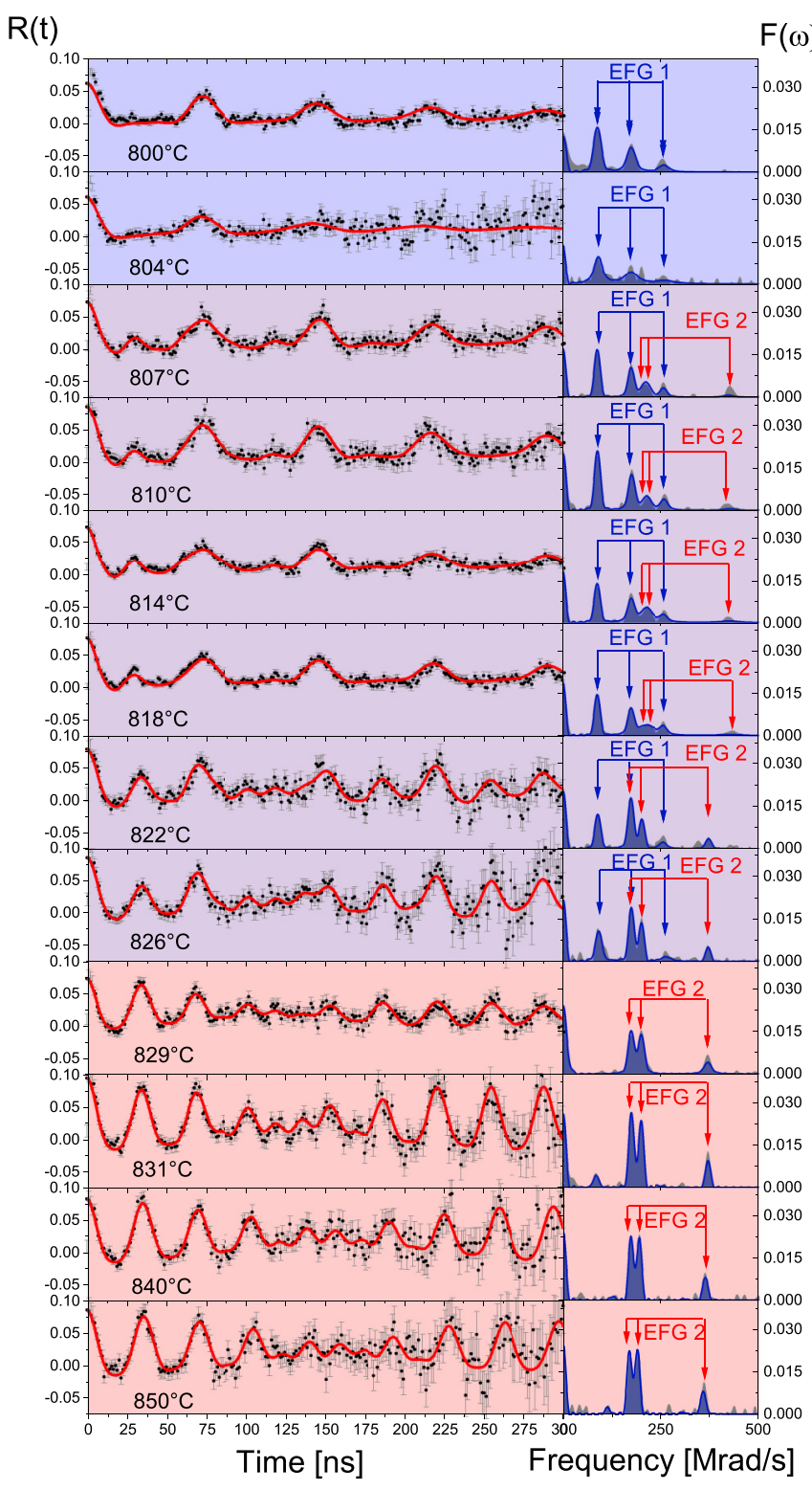

FIG. 4. TDPAC spectra with fitting functions in red and FFT showing the measured EFGs.

distorted, which changes the bond lengths of the coordinating atoms drastically. Regarding that, the coordination numbers mentioned here describe the number of nearest coordinating oxygen atoms which influence the environment of the cation most. The Bi ion in the $\alpha$-phase is located on a threefold rotation axis so symmetric by 3 -fold rotation (site symmetry 3 , trigonal point group). On the other hand, the site symmetry of the $\mathrm{Bi}$ position in the $\beta$-phase is $m$, which refers to a monoclinic point group, meaning that the $\mathrm{Bi}$ ion is located on a mirror reflection plane and so symmetric by mirror reflection. This change in symmetry is accompanied by a change in average coordination lengths from $d_{\mathrm{Bi}-\mathrm{O}_{\alpha}}=2.42 \AA$ [34] to $d_{\text {Bi- }{ }_{\beta}}=2.64 \AA$ [9], both visible in the PAC results as the following chapter will illustrate. The visualization of the single EFGs in Fig. 4 already hints towards the changes happening during the phase transition from the $R 3 c$ to the Pbnm phase as it shows a clear assignment of EFG1 to the $\alpha$-phase and

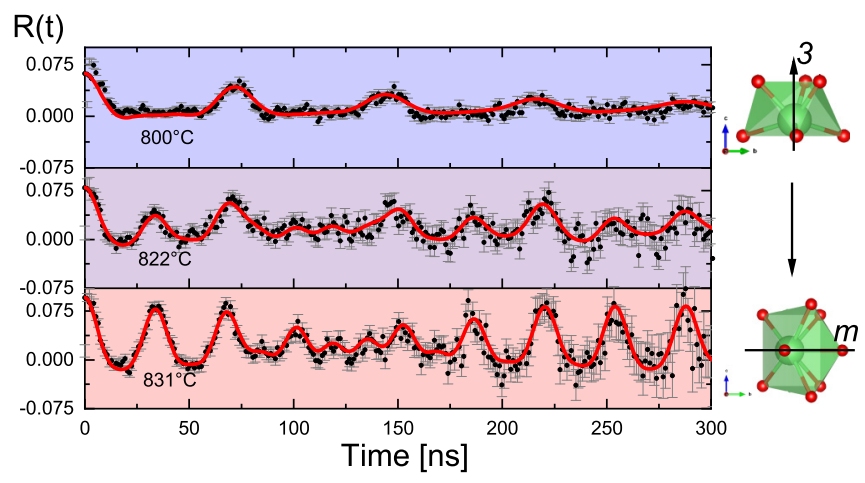

FIG. 5. Three distinctive PAC spectra showing single $\alpha$-phase on top, the disordered range with a combination of $\alpha$ and $\beta$ coordination in the middle, and pure $\beta$-phase in the bottom spectrum. Specific $\mathrm{Bi}(\mathrm{Cd})-\mathrm{O}$ polyhedra with symmetry elements to the right.

of EFG2 to the $\beta$-phase. The data acquisition as well as the fitting and the calculation of the experimental EFG were done with the GFIT 19 software package, a modified version of the NNFIT software package $[35,36]$. Hyperfine parameters of all fits were calculated and can be found in Table II. The higher number of near neighboring oxygen atoms in the surrounding of the cadmium probe atom in eight-fold coordination $(\beta$ phase) leads to an increased quadrupole interaction frequency with respect to the 6-fold coordination in the $\alpha$-phase which can be seen in Fig. 5. Up to a measurement temperature of $804^{\circ} \mathrm{C}$, only the lower frequency EFG $\left(\omega_{0} \sim 86 \mathrm{Mrad} / \mathrm{s}\right)$ is present. Compared with the simulation results listed in Table I, we associate this field gradient (EFG1) to $\alpha$-BFO in ferromagnetic order with $\mathrm{Cd} @ \mathrm{Bi}$. The measured asymmetry parameter $\eta$ also matches the simulated value reasonably well. As is visible on the FFT of EFG1 in Fig. 4, the low value of $\eta$ close to zero leads to a $\omega_{1}: \omega_{2}: \omega_{3}$ ratio of $1: 2: 3$ (compare to Fig. 3). Starting at $807^{\circ} \mathrm{C}$, also the high frequency field gradient $\left(\omega_{0} \sim 100-120 \mathrm{Mrad} / \mathrm{s}\right)$ appears. This EFG is linked to $\beta$-BFO in ferromagnetic order (compare with Table I), also with Cd@Bi. The mismatch of the measured and the simulated value of $\eta$ is considered less important than the matching values of $\omega_{0}\left(V_{z z}\right)$ [37]. Above $826^{\circ} \mathrm{C}$, only the high frequency field gradient (EFG2, $\beta$-phase) is detected. As seen in Fig. 6, the values of $\omega_{0}$ assigned to the $\alpha$-phase are practically constant throughout the temperature increase. On the other hand, the quadrupole frequency of EFG2 which is assigned to the $\beta$-phase shows a decrease from $\omega_{0}=$ $123.67 \mathrm{Mrad} / \mathrm{s}$ at $818^{\circ} \mathrm{C}$ to $\omega_{0}=110.05 \mathrm{Mrad} / \mathrm{s}$ at $822^{\circ} \mathrm{C}$. This can also be seen in the FFT functions in Fig. 4 as EFG2 clearly changes between $818^{\circ} \mathrm{C}$ and $822^{\circ} \mathrm{C}$. This feature is the indication for the phase transition. The discordant values of $\omega_{0}$ of EFG2 right before that decrease indicate the following: With increasing temperature and the approaching of the phase transition temperature, local coordination disordering of the Bi-site, which below $820^{\circ} \mathrm{C}$ is still in its 6-fold $\alpha$-phase coordination, appears. This means that a pseudo $m$-symmetry coordination, which actually belongs to the not yet existing $\beta$-phase, starts to appear on the 3 -fold-symmetry site of the $\mathrm{Bi}$ ion. This disordered coordination distorts the EFG and leads to the high values of $\omega_{0}$. The second parameter to distinguish between the $\alpha$ and the $\beta$ phases is the asymmetry 
TABLE II. Experimental PAC values of hyperfine parameters.

\begin{tabular}{|c|c|c|c|c|c|c|c|c|}
\hline \multirow[b]{2}{*}{ Temp. $\left[{ }^{\circ} \mathrm{C}\right]$} & \multicolumn{4}{|c|}{$\alpha$-phase } & \multicolumn{4}{|c|}{$\beta$-phase } \\
\hline & $\omega_{0}^{\mathrm{PAC}}[\mathrm{Mrad} / \mathrm{s}]$ & $\eta^{\mathrm{PAC}}[1]$ & $\delta^{\mathrm{PAC}}[\%]$ & $\%$ & $\omega_{0}^{\mathrm{PAC}}[\mathrm{Mrad} / \mathrm{s}]$ & $\eta^{\mathrm{PAC}}[1]$ & $\delta^{\mathrm{PAC}}[\%]$ & $\%$ \\
\hline 500 & $106(6)$ & $0.44(7)$ & $30(7)$ & 100 & & & & \\
\hline 650 & $88.5(3)$ & $0.14(2)$ & $4.6(4)$ & 100 & & & & \\
\hline 703 & $87(1)$ & $0.23(3)$ & $10(1)$ & 100 & & & & \\
\hline 776 & $86.8(5)$ & $0.16(2)$ & $10(1)$ & 100 & & & & \\
\hline 804 & $87.3(5)$ & $0.16(4)$ & $10.3(6)$ & 100 & & & & \\
\hline 807 & $86.5(2)$ & $0.08(1)$ & $2.0(3)$ & $78(5)$ & $122(0)$ & $0.92(1)$ & $0.2(7)$ & $22(2)$ \\
\hline 810 & $86.8(2)$ & $0.02(2)$ & $2.6(3)$ & $79(9)$ & $120.54(0)$ & $0.99(4)$ & $4(1)$ & $21(3)$ \\
\hline 814 & $86.5(2)$ & $0.07(2)$ & $3.3(3)$ & $65(6)$ & $121.14(0)$ & $0.99(2)$ & $5.6(7)$ & $35(2)$ \\
\hline 818 & $86.3(2)$ & $0.08(1)$ & $2.2(2)$ & $61(2)$ & $123.67(0)$ & $0.90(3)$ & $10(1)$ & $39(2)$ \\
\hline 822 & $85.4(5)$ & $0.16(1)$ & $2.5(1)$ & $50(6)$ & $110.05(0)$ & $0.83(0)$ & $0.7(3)$ & $50(6)$ \\
\hline 850 & & & & & $105.3(3)$ & $0.86(0)$ & $0.5(2)$ & 100 \\
\hline
\end{tabular}

parameter $\eta$. As clearly seen in Fig. 7, the $\alpha$-phase appears in a rather symmetric setting $\left(\eta_{\alpha} \sim 0.1\right)$ compared to the $\beta$ phase $\left(\eta_{\beta} \sim 0.9\right)$. There is a slight change of the asymmetry parameter in EFG2 between $814^{\circ} \mathrm{C}$ and $822^{\circ} \mathrm{C}$ as the value drops from $\eta_{814}=0.996$ to $\eta_{822}=0.828$. This dynamic is linked to the coordination disordering described before. The surrounding of the probe atom thus experiences a noticeable structural change in this temperature region. An indication for these dynamics are the slightly increasing values of damping $\delta$ in the $\beta$-EFG between $807^{\circ} \mathrm{C}$ and $818^{\circ} \mathrm{C}$ (Fig. 8), as with the increasing number of disordered $\mathrm{Bi}$-coordination sites the EFG distribution width broadens. The damping $\delta$, shown in Fig. 8, is generally low at all measurements, indicating a rather narrow EFG distribution and thus allows an accurate analysis. It also shows that the change in EFG due to fluctuations is low.

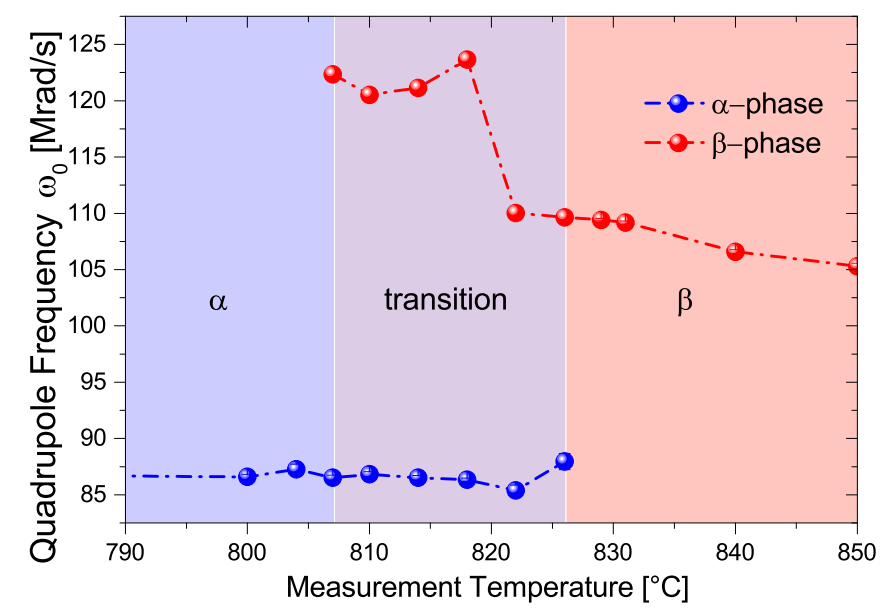

FIG. 6. Temperature dependence of the quadrupole interaction frequency $\omega_{0}$. The blue area shows the rhombohedral $\alpha$-phase region and the red area the orthorhombic $\beta$-phase region with the area of disordered coordination in purple in between. If no errorbars are visible, the symmetrical error is smaller than the marker size.
Thus, the crystal structure itself changes likely due to longer range interactions. The disordering of the $\mathrm{Bi}$-sites and the following changes in coordination are an independent effect, which are only visible locally. The visible changes in damping $\delta$, even though they are small, have a significant meaning. EFG1 by comparison shows a peak in damping at $804^{\circ} \mathrm{C}$. This feature indicates the emergence of the $\beta$-phase EFG, caused by the mentioned pseudo $m$-symmetry coordinations, which is already present at the next measurement point at $807^{\circ} \mathrm{C}$. The increasing $\delta$-value between $807^{\circ} \mathrm{C}$ and $818^{\circ} \mathrm{C}$ in EFG2 was already addressed before. At $822^{\circ} \mathrm{C}$, the damping again drops to a value of $\delta_{822}{ }^{\circ} \mathrm{C}=0.68(27) \%$. This indicates that the phase transition is completed. From now on, the $\mathrm{Bi}$ ion sits on the mentioned site with $m$-symmetry in an eightfold coordination. EFG1 exists up to a temperature of $826^{\circ} \mathrm{C}$, where the last

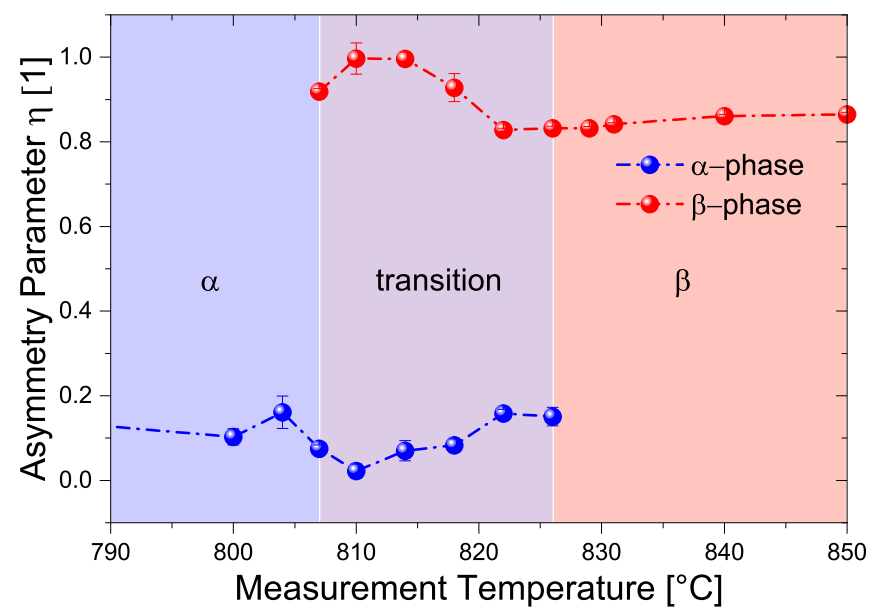

FIG. 7. Temperature dependence of the asymmetry parameter $\eta$. The blue area shows the rhombohedral $\alpha$-phase region and the red area the orthorhombic $\beta$-phase region with the area of disordered coordination in purple in between. If no errorbars are visible, the symmetrical error is smaller than the marker size. 


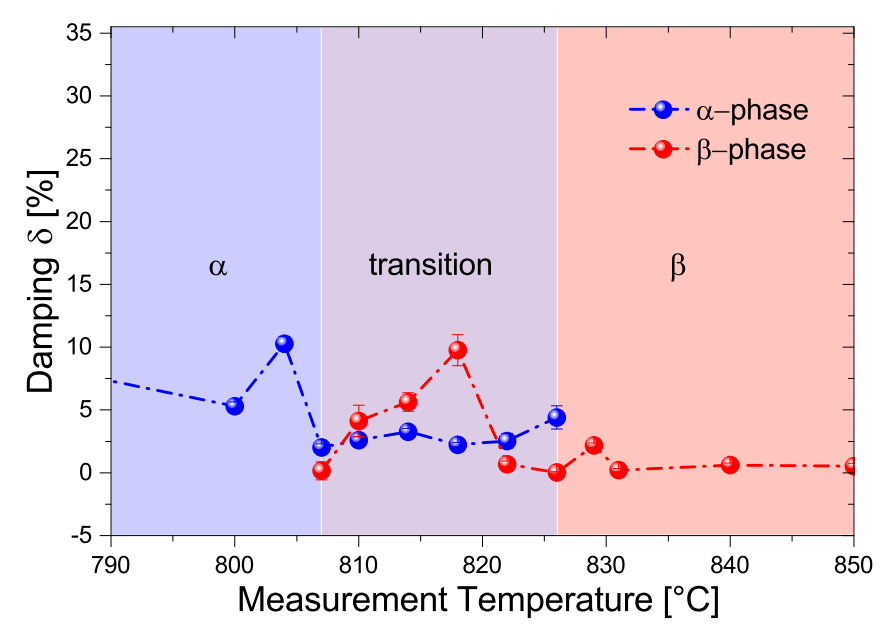

FIG. 8. Temperature dependence of the damping parameter $\delta$. The blue area shows the rhombohedral $\alpha$-phase region and the red area the orthorhombic $\beta$-phase region with the area of disordered coordination in purple in between. If no errorbars are visible, the symmetrical error is smaller than the marker size.

remaining pseudocoordinates with 3 -fold-symmetry vanish. As can be seen in Fig. 9, the fraction of EFG2 is increasing with increasing temperature while the fraction of EFG1 decreases. This, once again, underlines the dynamics of the coordination change as the ${ }^{111 m} \mathrm{Cd}$ probe ion forecasts the phase transition. An alternative explanation which is not in contradiction with our measurement results was given by Prosandeev et al. [38] with a polytype phase that acts as an intermediate structure between the $R 3 c \alpha$-phase and the Pbnm $\beta$-phase. This putative transition structure is designated "nano-twinned" in the work of Prosandeev et al. PAC data, as mentioned, allow a very specific, local picture of the closest environment of the probe atom. Thus, the resulting polytype structure, with a period length of six lattice constants along [001] cannot be measured as a unique, independent EFG but

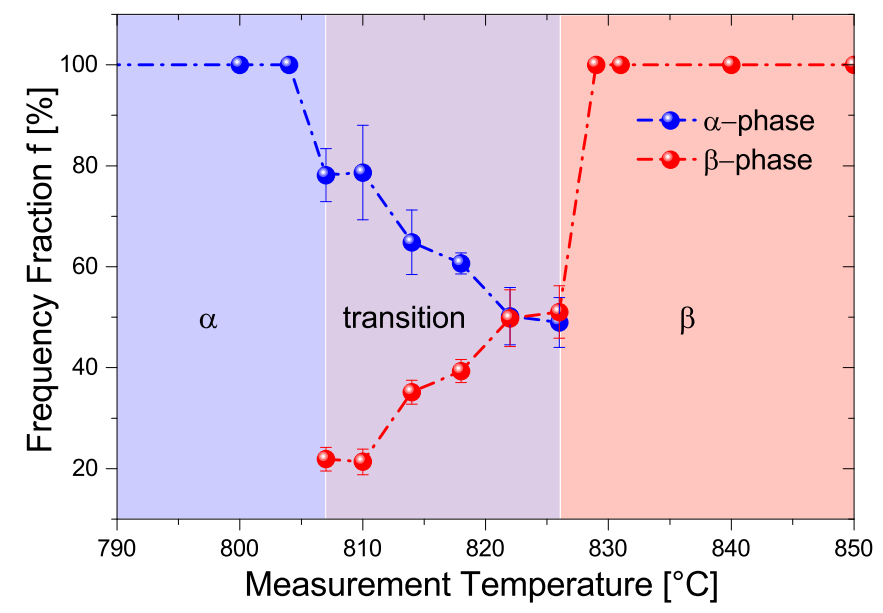

FIG. 9. Temperature dependence of the frequency fraction $f$. The blue area shows the rhombohedral $\alpha$-phase region and the red area the orthorhombic $\beta$-phase region with the area of disordered coordination in purple in between. If no errorbars are visible, the symmetrical error is smaller than the marker size. can be interpreted through the coincident appearance of EFG1 and EFG2. In contrast to the predictions of Prosandeev et al., the observed transition structure is stable only in a $19 \mathrm{~K}$ as opposed to a $250 \mathrm{~K}$ region. Considering this, the phase transition can be explained as a combination of two independent phase transitions following each other. The visible transition range around the $\alpha-\beta$ phase transition in BFO to our knowledge is a novel result. This range is thus not only a response to the substitutional $\mathrm{Cd}$ cations but also to global crystal symmetry change: On the one hand, crystal defects, such as the $\mathrm{Cd}$ substitution in BFO, lead to strong distortions in their closest vicinity which also might induce a delayed or accelerated phase transition, at least on a local point of view. The local defect induced broadening of the transition range can only be measured with PAC and not with XRD. On the other hand, the XRD data of pure BFO show a hysteresis around $T_{c}$ (see below), which is smaller but with a width of around $8 \mathrm{~K}$ accounts for roughly $45 \%$ of the transition range width measured with PAC. In consideration of the mentioned details, we assume that there is a hysteresis of the long-range order parameter. The increase of the frequency fraction attributed to the $\beta$-phase in Fig. 9 can be interpreted as a phase transition of the second order. Such a second-order phase transition could either mean an increase of the fraction of the polytypes predicted by Prosandeev et al. or a continuous decrease of the periodicity of the repetition period of the stacking, i.e., an increase in $\beta$-BFO like coordination.

\section{Probe site assignment}

Site assignment for tracer ions is sometimes a difficult task. We here collect arguments for $A$ or $B$ site substitution. DFT simulations, which are only strictly valid at a temperature of $0 \mathrm{~K}$, might to some extent be uncertain at our measurement temperatures. A comparison of the ionic radii of the involved elements strongly suggests a substitution of the Bi site through the $\mathrm{Cd}$ ion. Depending on the coordination number $(\mathrm{CN})$, the ionic radii of all ions vary as summarized in Table III. Bi as well as the Fe cations are assumed to be in a 6-fold coordination in the $\alpha$-phase, with their effective ionic radii of $1.02 \AA$ and $0.55 \AA$ (low spin)/0.645 $\AA$ (high spin), respectively. Thus, the value of a 6 -fold coordination of $\mathrm{Cd}^{2+}$ was taken for comparison [9]. The 6-fold effective ion radius of $\mathrm{Cd}$ with $0.95 \AA$ is much closer to the radius of the $\mathrm{Bi}$ ion than to the $\mathrm{Fe}$ ion, which is the most important indication for the substitution

TABLE III. Effective ion radii of $\mathrm{Cd}^{2+}, \mathrm{Bi}^{3+}$, and $\mathrm{Fe}^{3+}$ ions in $\AA$ for different coordination numbers $\mathrm{CN}$ [39].

\begin{tabular}{lccccc}
\hline \hline \multirow{2}{*}{} & \multicolumn{5}{c}{ Ion } \\
\cline { 2 - 6 } & $\mathrm{Cd}^{2+}$ & $\mathrm{Bi}^{3+}$ & $\mathrm{Fe}^{3+}$ & $\mathrm{In}^{3+}$ & $\mathrm{Mn}^{3+}$ \\
\hline $\mathrm{IV}$ & 0.84 & - & 0.49 & - & - \\
V & 0.87 & 0.99 & - & - & 0.58 \\
VI & 0.95 & 1.02 & $0.55(\mathrm{LS})$ & 0.79 & $0.58(\mathrm{LS})$ \\
& & & $0.645(\mathrm{HS})$ & & 0.65 (HS) \\
VII & 1 & - & - & & - \\
VIII & 1.07 & 1.11 & - & 0.923 & - \\
XII & 1.31 & - & - & & \\
\hline \hline
\end{tabular}


at the $A$-site [39]. A substitution of the Fe cation would necessarily lead to a significant distortion of the unit cell, possibly followed by an asymmetry parameter different from zero. A counterargument would be that ferroelectricity in $\mathrm{BiFeO}_{3}$ is mainly due to the lone pair polarization of the $\mathrm{Bi}$ ion. Thus, Cd substituting Bi must experience the local effect of changes in polarization in its field gradient. So, the EFG should change also before the curie point itself ( $\alpha$ to $\beta$ phase transition) is reached. Landau theory predicts a moderate change of the order parameter just beneath such a strong first-order phase transition [40]. So unlike in second-order phase transitions, where a large change in $V_{z z}$ would have to precede the phase transition, the jump in order parameter at the first-order phase transition temperature allows for only moderate changes just beneath $T_{c}$. We observe practically no change in $V_{z z}$ beneath the phase transition temperature and assign this to the very limited temperature range we investigate. In this range no change is within the error bar of analysis with a "moderate" change which would be a few percent only as we know from other data on $\mathrm{BiFeO}_{3}$. The ${ }^{111 m} \mathrm{Cd}$ probe site substitution was also a point of discussion in rare-earth orthochromite ceramics. Comparisons with ${ }^{111} \operatorname{In}\left({ }^{111} \mathrm{Cd}\right)$ PAC measurements done on rare-earth orthoferrites lead to the assumption that in the particular case of $\mathrm{SmCrO}_{3}$ the ${ }^{111 m} \mathrm{Cd}$ probe also occupies the $A$-site [41]. The ${ }^{111} \operatorname{In}\left({ }^{111} \mathrm{Cd}\right)$ probe used to examine the rareearth orthoferrites $\left(\mathrm{RFeO}_{3}, \mathrm{REO}\right)$ occupies both sites, leading to a low frequency EFG describing the Fe-occupancy and a high frequency EFG describing the $R$-occupancy [42]. As seen in Table III, ${ }^{111}$ In has a significantly smaller ionic radius (0.79 $\AA$ in 6-fold coordination) than ${ }^{111 m} \mathrm{Cd}$, which possibly leads to an occupation of both sites. Rearick et al. [42] also mentioned that the ${ }^{111}$ In probe primarily substitutes into the rare-earth sites in the heavier REOs. Though bismuth is not a rare-earth element, an $A$-site occupation is more likely as $\mathrm{Bi}$ is heavier than all the rare earth elements. Further literature affirming the $A$-site substitution are the works of Bellakki [43], which addressed Cd-doped BFO and Ishaq et al. [44], which treats $\mathrm{Cd}-\mathrm{Mn}$ doped BFO. Bellakki states that $\mathrm{Cd}$ substitutes only the Bi cation. Ishaq et al. described that $\mathrm{Cd}$ substitutes the Bi site whereas Mn substitutes the Fe site. Reconsidering the ionic radii of $\mathrm{Cd}$ and $\mathrm{Mn}$ (Table III) this is expected as the value of $\mathrm{Mn}$ is significantly closer to the one of $\mathrm{Fe}$ than the ionic radius of $\mathrm{Cd}$ which is similar to $\mathrm{Bi}$. Sato et al. also describe ${ }^{111 m} \mathrm{Cd}$ as substituting the $R$ and $A$ sites in doped perovskite manganese oxides $\left(R_{1-x} \mathrm{~A}_{x} \mathrm{MnO}_{3}\right)$ [45]. As in our work only one EFG is assigned to the $\alpha$-phase, $A$-site occupancy is what we conclude from these arguments.

\section{XRD results}

The powder diffraction measurements were performed in air using a PANalytical X'Pert Pro MPD $\theta-\theta$ diffractometer equipped with an Anton Paar HTK 1200 high temperature chamber and an $X^{\prime}$ celerator 128 channel detector. Intensity data were collected in the $2 \theta=20^{\circ}-70^{\circ}$ range with a scan length of $2.122^{\circ}$ with $55.2 \mathrm{~s}$ exposure per scan length resulting in overall measurement times of about 45 minutes. Data were converted into step size bins of $0.0084^{\circ}$. This measurement routine was used to determine the overall phase composition of the samples at different temperature points around

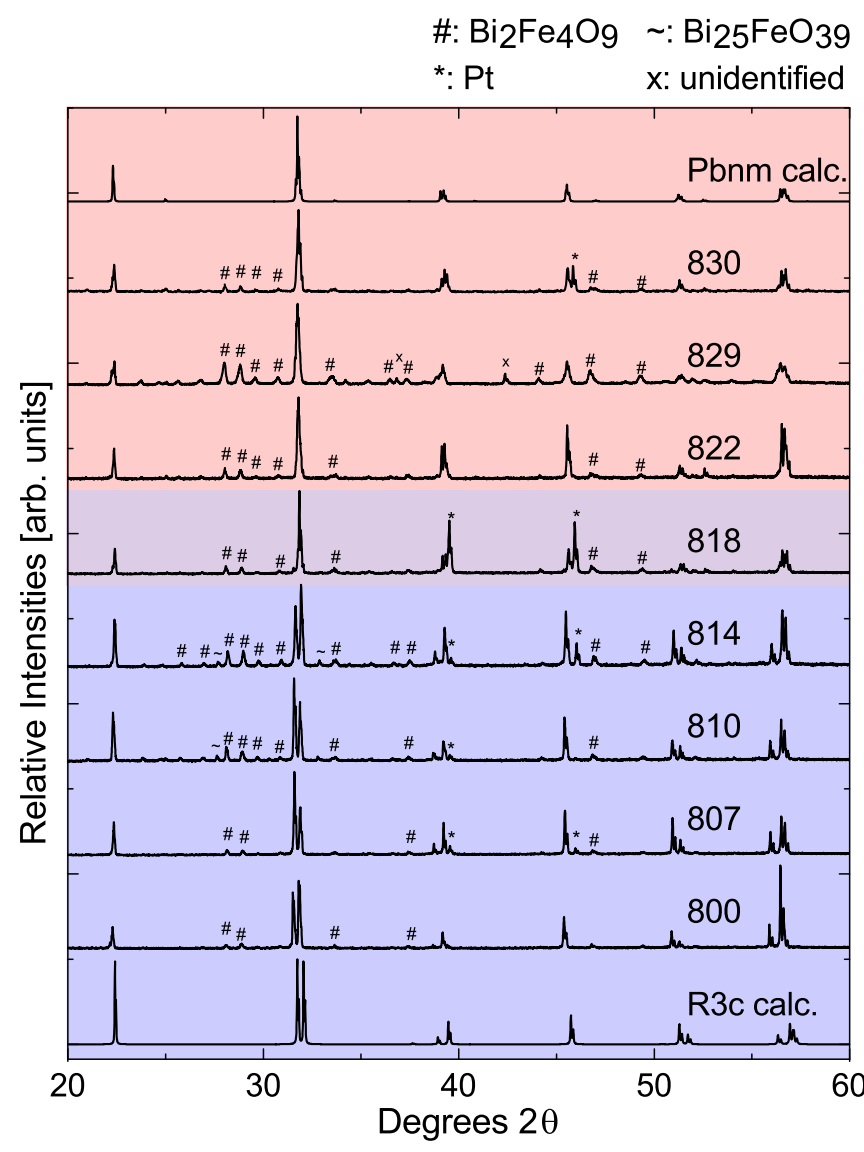

FIG. 10. Temperature dependence of x-ray diffractograms measured between $20^{\circ}-70^{\circ}$ (graphs are only shown up to $60^{\circ}$ as no information gain is achieved from reflections above $60^{\circ}$ ) at increasing temperatures around the phase transition temperature. The top and bottom graphs show calculated diffractograms of BFOs $R 3 c$ and Pbnm structure, respectively. The intensities are normalized to the highest intensity.

the presumed Curie temperature as suggested by the PAC measurements. Sweep measurements covering the $2 \theta$-range between $30^{\circ}$ and $35^{\circ}$ were done to determine the exact phase transition temperature. Samples were ground and the powder was placed on platinum sample holders to avoid reactions with standard sample holders such as Si single crystals, quartz or Macor. Figure 10 shows XRD diffractograms of BFO, measured at temperatures between $800^{\circ} \mathrm{C}$ and $830^{\circ} \mathrm{C}$ as well as XRD patterns calculated from crystallographic information files (CIFs) for $\alpha$-BFO with $R 3 c$ and $\beta$-BFO with Pbnm symmetry. The structural data given in the CIF were also used for the simulations described in Sec. III A. This gives a first impression of the conformance of the results of PAC and XRD measurements. The calculated diffractogram for the $\alpha$ phase coincides perfectly with the measured data up to $814^{\circ} \mathrm{C}$ (Fig. 10), thus this temperature region is considered to represent the single $\alpha$-phase. Rietveld refinement backs this as the $\beta$-phase does not appear below $818^{\circ} \mathrm{C}$. This temperature can be considered as the phase transition point of a first-order phase transition, as $\alpha$ and $\beta$ phase are present simultaneously. This diffractogram is mostly consistent with the $\beta$-phase. However, minor amounts of the $\alpha$-phase are still observed for example by the not completely vanished 104 reflection at 


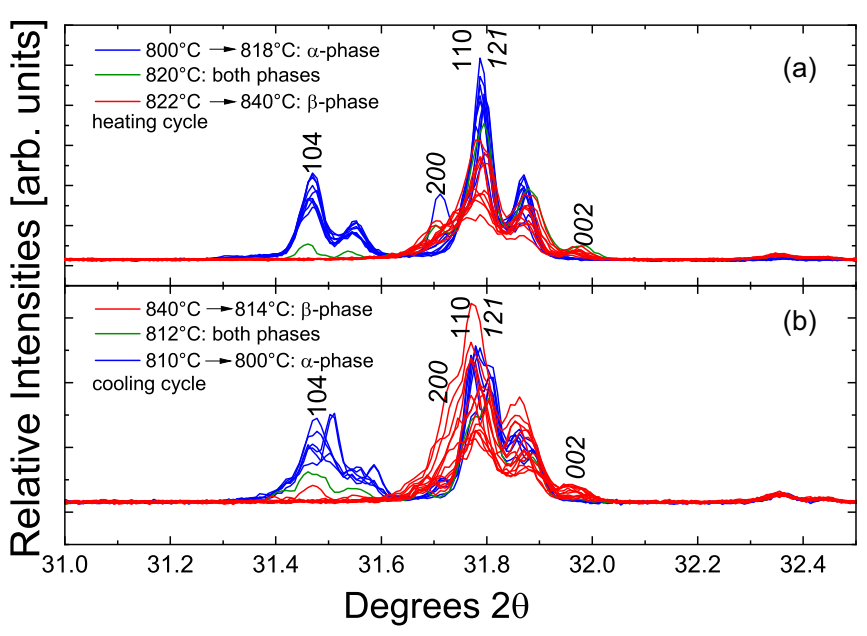

FIG. 11. Reflection behavior at the phase transition. $\alpha$-phase in blue, mixed phase in green, and $\beta$-phase in red. Reflection descriptions of the $\beta$-phase are italic. (a) The top diffractogram shows the reflection merging during the heating cycle, (b) the lower diffractogram shows the reflection splitting during the cooling cycle.

$2 \theta=31.5^{\circ}$, which is most likely caused by a temperature gradient. Rietveld refinement results in a $\alpha$-BFO fraction of ca. $8 \%$. The additional reflections appearing in the diffractograms are either Pt reflections from the sample holder (marked with *) or of the known decomposition products $\mathrm{Bi}_{2} \mathrm{Fe}_{4} \mathrm{O}_{9}$ (\#) and $\mathrm{Bi}_{25} \mathrm{FeO}_{39}(\sim)$ as $\mathrm{BFO}$ is metastable at elevated temperatures [46]. The two reflections labelled with $\mathrm{x}$ were not identified but could result from sample contamination during the measurements. As mentioned, the phase transition was also analyzed through sweep measurements in a small angle range, determined to ascertain the exact phase transition temperature as well as the hysteresis around the transition point. A suitable reflection for this examination was found to be the 104-2 $\overline{1} 0$ doublet of $\alpha$-BFO at approximately $2 \theta=31.5^{\circ}$ and $2 \theta=32^{\circ}$, respectively, which merges to a narrow reflection triplet of 020,112 , and 200 of the orthorhombic $\beta$-phase when the structural phase transition occurs (see Fig. 11). The sweep was measured with temperature steps of $2 \mathrm{~K}$ between $800^{\circ} \mathrm{C}$ and $840^{\circ} \mathrm{C}$. Up to a temperature of $818^{\circ} \mathrm{C}$, the diffractogram of the heating cycle shows clear evidence of only the $\alpha$-phase being present. In the $820^{\circ} \mathrm{C}$ dataset, shown in green, the diffractogram shows a distinctly weaker intensity at the 104 reflection and also an attenuated intensity of the $2 \overline{1} 0$ reflection, leading to the conclusion that the $\alpha$-phase is vanishing, representing the region of coexistence of both phases. In all datasets acquired above $820^{\circ} \mathrm{C}$, shown in red, only the mentioned triplet of the $\beta$-phase is present. A hysteresis is visible because the $\alpha$-phase starts to appear only at $812^{\circ} \mathrm{C}$ during the cooling cycle. Rietveld refinement of the sweep measurements allows a coarse estimation of the available phase fractions which are shown for the heating cycle in red and the cooling cycle in blue in Fig. 12. For the data line in red in Fig. 11(b), which shows a very first appearance of the 104 reflection $\left(814^{\circ} \mathrm{C}\right)$, the Rietveld refinement estimated a zero percent $\alpha$-phase fraction. Again, through the very narrow angular range of the sweep measurement, an exact refinement is difficult but the results are

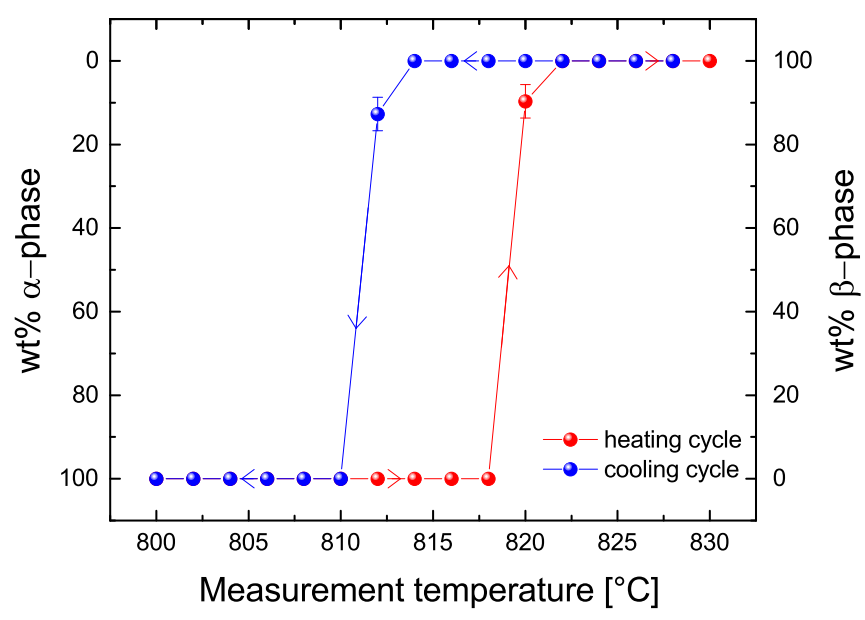

FIG. 12. Temperature development of the Rietveld refinement phase fractions given in weight percent showing a clear hysteresis. The heating cycle is depicted in red and the cooling cycle in blue.

specific enough to describe the appearing hysteresis of around $8 \mathrm{~K}$ (Fig. 12). The polytype transition structure described by Prosandeev et al. could not be evidenced by XRD data. This is not unusual since such polytypes often cause tiny satellites, which cannot be seen in standard powder diffraction experiments. Sometimes such phase transitions between a basic and a superstructure are reflected in a change of cell parameters. However, we did not observe any anomaly in the thermal expansion of the $\alpha$-phase close to the phase transition temperature. The temperature difference of $2 \mathrm{~K}$ between the phase transition temperature observed in these sweep measurements in the heating cycle $\left(820^{\circ} \mathrm{C}\right)$ and the measurements covering the angle between $20^{\circ}$ and $70^{\circ}\left(818^{\circ} \mathrm{C}\right)$ can be explained through uncertainties of the temperature measurement in the high temperature chamber during the different single experiments. Reflections of the ternary mullite $\mathrm{Bi}_{2} \mathrm{Fe}_{4} \mathrm{O}_{9}$ and the selenite $\mathrm{Bi}_{25} \mathrm{FeO}_{39}$ phases are visible in all measured diffractograms (Fig. 10). These decomposition products, which also form under vacuum and inert gas, were not present in the samples before they were subjected to the measurements at high temperatures. In fact, comparisons of diffractograms shown in Fig. 13 recorded at temperatures between $600^{\circ} \mathrm{C}$ and $800^{\circ} \mathrm{C}$ show that the impurity phases start to appear around about $650^{\circ} \mathrm{C}$ with $\mathrm{Bi}_{25} \mathrm{FeO}_{39}$ already being present at about $650^{\circ} \mathrm{C}$, whereas $\mathrm{Bi}_{2} \mathrm{Fe}_{4} \mathrm{O}_{9}$ appears slightly later around $700^{\circ} \mathrm{C}-720^{\circ} \mathrm{C}$. Obviously these secondary phases are more stable with respect to pure $\mathrm{BFO}$ in this temperature range [47]. $\mathrm{BFO}$ decomposes into the mullite and selenite phases following the reaction $49 \mathrm{BiFeO}_{3} \rightleftarrows 12 \mathrm{Bi}_{2} \mathrm{Fe}_{4} \mathrm{O}_{9}+\mathrm{Bi}_{25} \mathrm{FeO}_{39}$, leading to significantly higher amounts of the mullite component, which is supported by our XRD results following Rietveld refinement [48]. Besides the measurement temperature and time, also the purity of the samples has a big impact on the formation rate of the ternary phases [18]. As the maximum measured temperature in our research was $840^{\circ} \mathrm{C}$, a halting of the decomposition processes as well as the reverse reaction from the secondary phases back to $\mathrm{BiFeO}_{3}$ as proposed by Selbach et al. [47] could not be confirmed. However, Fig. 13 shows that at $840^{\circ} \mathrm{C}$ the 013 reflection of $\mathrm{Bi}_{25} \mathrm{FeO}_{39}$ is 


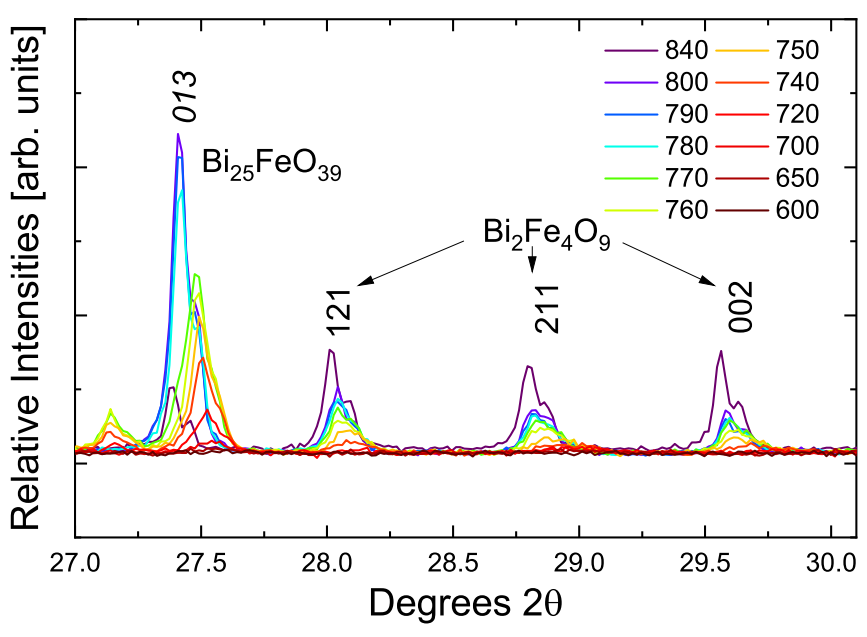

FIG. 13. Peak growth of ternary phases $\mathrm{Bi}_{25} \mathrm{FeO}_{39}$ (italic) and $\mathrm{Bi}_{2} \mathrm{Fe}_{4} \mathrm{O}_{9}$ with temperature. The color gradient corresponds to the temperature change.

significantly weaker compared to the peak intensities at lower temperatures, indicating the beginning of a reduction of the presence of this component. On the other hand, all peaks of $\mathrm{Bi}_{2} \mathrm{Fe}_{4} \mathrm{O}_{9}$ show stronger intensities even at $840^{\circ} \mathrm{C}$. Thus, the thermodynamic stabilities of the two ternary phases might not be equal. The simulation results listed in Table I were done to try a fitting of the PAC results including phase fractions of $\mathrm{Bi}_{2} \mathrm{Fe}_{4} \mathrm{O}_{9}$ with the calculated parameters. However, no phase fractions of the mullite phase were present in the PAC spectra. The appearance of the ternary phases in the XRD measurements, which were conducted after the PAC measurements, could be explained through the second heating process at the XRD measurements which leads to an increase of fractions of those phases. Visible ternary phase fractions in samples which were not exposed to a prior PAC measurement might be caused through the vigorously larger surface area of powdered samples in comparison to bulk samples used during the PAC measurements, easing the volatility of $\mathrm{Bi}$ and thus the building of the decomposition products.

\section{CONCLUSION}

The ferro to paraelectric phase transition of multiferroic $\mathrm{BiFeO}_{3}$ was investigated through two fundamentally different experimental methods. PAC, which is an extremely sensitive method probing the sample on an atomic scale and XRD. The results of both experimental methods agree with a phase transition from rhombohedral $\alpha$-BFO in $R 3 c$ setting to orthorhombic $\beta$-BFO with its Pbnm space group. The assumption of the Bi-site substitution by the ${ }^{111 m} \mathrm{Cd}$ probe is confirmed by crystal chemical assignment (ion radii) as well as by our DFT and PAC results. Following the PAC results, the first-order phase transition is forecast by the $\mathrm{Cd}$ probe ion as the preceding symmetry change of Bi-coordination is sensed. The phase transition point at $820^{\circ} \mathrm{C}$ is visible through a drop in quadrupole interaction frequency $\omega_{0}$ which indicates the site symmetry change of the Bi position. The discordant values of $\omega_{0}$ and $\eta$ within the temperature interval of $807^{\circ} \mathrm{C}$ and $818^{\circ} \mathrm{C}$ could be explained by the gradual formation of a polytype transition structure or by growing disorder. In the former case, the alpha-beta phase transition would actually be a combination of a second order followed by a first-order phase transition. The first could not be evidenced in XRD. Thus, it is shown that both methods are complementary. The transition range in PAC and thermal hysteresis measured with XRD suggests a change of the long-range order parameter around the phase transition point. A broadening of the transition range at the phase transition as a result of the disturbed local environment around the probe ion itself is visible through the wider range at the PAC measurements compared to the XRD results. The visibility of the mentioned possible dynamics during the phase transition in the results over a temperature range of $19 \mathrm{~K}$ again underlines the precision of PAC measurements at high temperatures, which transcends the performances of other nuclear measurement methods like Mössbauer or nuclear magnetic resonance spectroscopy in this temperature range. This is also a very fundamental result as we have proof of the local change of the order parameter caused by defects, which was often assumed in literature before [4]. Following the XRD results, the phase transition happens at a transition temperature of $818^{\circ} \mathrm{C}$ as this is the only temperature point where both phases appear simultaneously. Indications of a polytype transition structure could not be found in our XRD data but its existence cannot be excluded. The appearance of the ternary phases $\mathrm{Bi}_{2} \mathrm{Fe}_{4} \mathrm{O}_{9}$ and $\mathrm{Bi}_{25} \mathrm{FeO}_{39}$ was observed. They only arise in powdered samples of high surface area as were used for XRD and are not contained in any PAC data.

\section{ACKNOWLEDGMENTS}

We acknowledge the financial support received from the Federal Ministry of Education and Research (BMBF) through Grants No. 05K16PGA and No. 05K16SI1. We also acknowledge the support of all the technical teams at ISOLDE for their excellent work in delivering high-quality beams for TDPAC measurements. We acknowledge the support of the European Union's Horizon 2020 Framework research and innovation program under Grant Agreement No. 654002 (ENSAR2) given to the ISOLDE experiment IS647. We thank the Foundation for Science and Technology FCT, Portugal, via Grant No. CERN-FIS- PAR-0005-2017, and Dr. J. G. M. Correia for technical assistance during the beam time. Last but not least, we would like to express special thanks to the insightful thoughts and comments of the reviewers which have lead to an interesting discussion and revision of our paper.
[1] H. Schmid, Ferroelectrics 162, 317 (1994).

[2] N. A. Spaldin and M. Fiebig, Science 309, 391 (2005).

[3] H. Bea, M. Gajek, M. Bibes, and A. Barthélémy, J. Phys.: Condens. Matter 20, 434221 (2008).
[4] G. Catalan and J. F. Scott, Adv. Mater. 21, 2463 (2009).

[5] R. Haumont, J. Kreisel, P. Bouvier, and F. Hippert, Phys. Rev. B 73, 132101 (2006). 
[6] R. Palai, R. S. Katiyar, H. Schmid, P. Tissot, S. J. Clark, J. Robertson, S. A. T. Redfern, G. Catalan, and J. F. Scott, Phys. Rev. B 77, 014110 (2008).

[7] R. Haumont, I. A. Kornev, S. Lisenkov, L. Bellaiche, J. Kreisel, and B. Dkhil, Phys. Rev. B 78, 134108 (2008).

[8] I. A. Kornev, S. Lisenkov, R. Haumont, B. Dkhil, and L. Bellaiche, Phys. Rev. Lett. 99, 227602 (2007).

[9] D. C. Arnold, K. S. Knight, F. D. Morrison, and P. Lightfoot, Phys. Rev. Lett. 102, 027602 (2009).

[10] S. M. Selbach, T. Tybell, M.-A. Einarsrud, and T. Grande, Adv. Mater. 20, 3692 (2008).

[11] J. Wei, C. Wu, T. Yang, Z. Lv, Z. Xu, D. Wang, R. Haumont, and Z. Cheng, J. Phys. Chem. C 123, 4457 (2019).

[12] R. Catherall, W. Andreazza, M. Breitenfeldt, A. Dorsival, G. Focker, T. Gharsa, T. Giles, J. Grenard, F. Locci, P. Martins, S. Marzari, J. Schipper, A. Shornikov, and T. Stora, J. Phys. G 44, 094002 (2017).

[13] K. Johnston, J. Schell, J. Correia, M. Deicher, H. Gunnlaugsson, A. Fenta, E. David-Bosne, A. Costa, and D. C. Lupascu, J. Phys. G 44, 104001 (2017).

[14] J. Landers, S. Salamon, M. Escobar Castillo, D. Lupascu, and H. Wende, Nano Lett. 14, 6061 (2014).

[15] T. Karthik, T. D. Rao, A. Srinivas, and S. Asthana, arXiv:1206.5606.

[16] G. Arlt and H. Neumann, Ferroelectrics 87, 109 (1988).

[17] M. E. Lines and A. M. Glass, Principles and Applications of Ferroelectrics and Related Materials (Oxford University Press, New York, 2001).

[18] M. Valant, A.-K. Axelsson, and N. Alford, Chem. Mater. 19, 5431 (2007).

[19] J. Schell, M. Schmuck, I. Efe, T. Thanh Dang, J. Gonçalves, M. Escobar C., V. V. Shvartsman, A. R. Granadeiro Costa, U. Köster, R. Vianden, D. Noll, and C. Lupascu (unpublished).

[20] L. Volkova and D. Marinin, J. Supercond. Novel Magn. 24, 2161 (2011).

[21] J. Ziegler, SRIM-2013 software package, http://www.srim.org.

[22] M. Jäger, K. Iwig, and T. Butz, Rev. Sci. Instrum. 82, 065105 (2011).

[23] T. Wichert and E. Recknagel, Microscopic Methods in Metals (Springer, New York, 1986), pp. 317-364.

[24] J. Schell, P. Schaaf, and D. C. Lupascu, AIP Adv. 7, 105017 (2017).

[25] R. Nédélec, Rückstoßimplantation von $48 \mathrm{Cr}$ und erste PAC Messungen mit der Sonde 48Cr/48V, Ph.D. thesis, Diplomarbeit, Universität Bonn, 2002.

[26] E. Gerdau, J. Wolf, H. Winkler, and J. Braunsfurth, Proc. R. Soc. Lond. A 311, 197 (1969).
[27] P. Friedsam, Anorganische nichtmetallische Festkörper ohne Translationssymmetrie untersucht mit der Methode der gestörten $\gamma-\gamma$-Winkelkorrelation, Ph.D. thesis, Dissertation, Universität Bonn, 2000.

[28] J. Bartels, Fremdatome in Silizium-Kavitäten untersucht mit der gestörten Winkelkorrelation, Ph.D. thesis, Dissertation, Universität Bonn, 2002.

[29] J. Schell, D. C. Lupascu, J. G. Martins Correia, A. W. Carbonari, M. Deicher, M. B. Barbosa, R. D. Mansano, K. Johnston, I. S. Ribeiro, and ISOLDE collaboration, Hyperfine Interact. 238, 2 (2016).

[30] G. Kresse and J. Furthmüller, Phys. Rev. B 54, 11169 (1996).

[31] J. N. Gonçalves (private communication, 2018).

[32] H. Haas, S. P. Sauer, L. Hemmingsen, V. Kell, and P. W. Zhao, Europhys. Lett. 117, 62001 (2017).

[33] J. Gebhardt and A. M. Rappe, Phys. Rev. B 98, 125202 (2018).

[34] J.-M. Moreau, C. Michel, R. Gerson, and W. J. James, J. Phys. Chem. Solids 32, 1315 (1971).

[35] J. G. Correia for GFIT19 and N. P. Barradas for NNFIT programs (PAC MANual Lisbon 1992).

[36] N. P. Barradas, M. Rots, A. A. Melo, and J. C. Soares, Phys. Rev. B 47, 8763 (1993).

[37] M. Forker, Nucl. Instrum. Methods 106, 121 (1973).

[38] S. Prosandeev, D. Wang, W. Ren, J. Íñiguez, and L. Bellaiche, Adv. Funct. Mater. 23, 234 (2013).

[39] R. T. Shannon and C. T. Prewitt, Acta Crystallogr. Sect. B 25, 925 (1969).

[40] M. E. Lines and A. M. Glass, Principles and Applications of Ferroelectrics and Related Materials (Oxford University Press, New York, 2001).

[41] G. Oliveira, R. Teixeira, R. Moreira, J. Correia, J. Araújo, and A. Lopes, Sci. Rep. 10, 1 (2020).

[42] T. M. Rearick, G. L. Catchen, and J. M. Adams, Phys. Rev. B 48, 224 (1993).

[43] M. Bellakki and V. Manivannan, J. Mater. Sci. 45, 1137 (2010).

[44] B. Ishaq, G. Murtaza, S. Sharif, M. A. Khan, N. Akhtar, I. Will, M. Saleem, and S. M. Ramay, Results Phys. 6, 675 (2016).

[45] W. Sato, S. Komatsuda, H. Shimizu, R. Moriichi, S. Abe, S. Watanabe, S. Komatsu, T. Terai, S. Kawata, and Y. Ohkubo, Phys. Rev. B 100, 184111 (2019).

[46] A. Perejon, P. E. Sanchez-Jimenez, J. M. Criado, and L. A. Perez-Maqueda, J. Phys. Chem. 118, 26387 (2014).

[47] S. M. Selbach, M.-A. Einarsrud, and T. Grande, Chem. Mater. 21, 169 (2008).

[48] T. Carvalho and P. Tavares, Mater. Lett. 62, 3984 (2008). 